\title{
Electron Microscopic and Histopathological Study of the Overacting Inferior Oblique Muscle of the Human Eye
}

\author{
Mohamed K Kandeel ${ }^{1 *}$, MSc; Gamal S Desouki1, ${ }^{1}$ MD; Alaa El Deen S El Sagheer ${ }^{1}$ MD \\ and Amr A ELkamshoshy² MD
}

\section{* Corresponding Author: \\ Mohamed Kandeel \\ Profandeel@gmail.com}

Received for publication May 18, 2021; accepted July 9, 2021; published online July 12, 2021.

Copyright The Authors published by Al-Azhar University, Faculty of Medicine, Cairo, Egypt. Users have the right to read, download, copy, distribute, print, search, or link to the full texts of articles under the following conditions: Creative Commons Attribution-Share Alike 4.0 International Public License (CC $B Y-S A$ 4.0).

doi: 10.21608/aimj.2021.76587.1477

${ }^{l}$ Department of Anatomy and Embryology, Faculty of Medicine Al-Azhar University.Cairo, Egypt.

${ }^{2}$ Department of Ophthalmology, Faculty of Medicine, Alexandria University, Alexandria, Egypt.

\begin{abstract}
Background: Inferior oblique muscle overaction is a common disorder that has a correlation with the anatomical changes of the muscle itself. The changes differ according to the grade of the overaction.

Objective: To correlate between the histopathological alternations of the inferior oblique muscle and the different degrees of clinical overaction.

Patients and methods: Ten biopsies from inferior oblique muscles were taken during the surgery of strabismus after determining the clinical degree of the overaction. One muscle biopsy was taken during enucleation surgery and considered as a control. All biopsies were examined by light and transmission electron microscopes.

Results: By light microscopy, grade II and grade III inferior oblique overaction showed many histopathological findings as muscle fibers degeneration, variable size of muscle fibers, nerve bundles hypertrophy and fibro-fatty infiltrations. Grade III inferior oblique overaction showed fibrosis of perimysium and endomysium, mononuclear infiltration and fatty infiltration. By transmission electron microscopy the muscle fibers showed increased mitochondrial number and mitochondrial variability in shape and size, also showed increased vacuoles and fat droplets.

Conclusion: Histopathological alternations in the inferior oblique muscle correlate to the clinical grades of the muscle overaction.
\end{abstract}

Keywords: Inferior oblique; Overaction; Histopathological; Electron microscope.

\footnotetext{
Authorship: All authors have a substantial contribution to the article.

Disclosure: The authors have no financial interest to declare in relation to the content of this article. The Article Processing Charge was paid for by the authors.
}

\section{INTRODUCTION}

Inferior oblique muscle (IO) differs in its anatomical structure from other extraocular muscles (EOM), this made many researchers to find out the correlation between the muscle ultrastructural changes and the clinical effect on its action. ${ }^{1}$ The muscle arises from anterior part of the orbital floor near to the margin and inserts in the inferolateral quadrant of the sclera posterior to equator and in-between lateral and inferior rectus muscles. ${ }^{2}$ Overaction of the IO muscle produces elevated eye in adduction position. ${ }^{3}$

Overaction of the IO muscle can be divided into two types; primary which is of unknown mechanism, and secondary which occurs secondary to superior oblique muscle affection. ${ }^{4}$

As regard the clinical appearance of the IO overaction, it can be classified into three grades I, II and III. Grade I shows the IO overaction with extreme looking in up - in direction. Grade II the muscle overaction appears with the adduction. Grade III appears in normal primary gaze. ${ }^{5}$ The surgical method of taking the IO muscle biopsy is a routine step in the surgery itself. ${ }^{6}$ The EOMs are surrounded by epimysium. Perimysium surrounds the muscle cell fiber. Endomysium surrounds the muscle cell. Sacrolemma is the muscle cell plasma membrane and sacroplasm is the muscle cytoplasm. The sarcoplasm is composed of myofibers, peripherally located nuclei and other organelles. ${ }^{7}$ EOMs are composed two types of muscle fibers, the first one is Fibrillenstruktur or fast - twitch fibers and it's responsible for the fast movements of the eye. The second type is Felderstruktur and is responsible for slow muscle movements. ${ }^{8}$ Both types can be evaluated by electron microscopy. Examination by the light microscope reveals pale staining of fast twitch fibers with regular spacing between myofibers while dense staining appears in the slow - twitch fibers with irregular spacing inbetween. ${ }^{9}$ The current study aimed to correlate between the histopathological findings and electron microscopic 
structures of the IO muscle and the clinical effect on its action as regard the grades of the muscle overaction.

\section{PATIENTS AND METHODS}

The IO muscle samples involved in this study are ten muscles. The muscle samples collected during the routine IO myectomy surgery from six patients (eight biopsies from four patients and two biopsies from two patients). Every case was evaluated and examined prior to the surgery to determine the grade of overaction in Alex I care Ophthalmology center, Alexandria, Egypt. The muscle biopsies were categorized under two Grades, grade II and grade III. Grade I is not involved in the study as myectomy is not a line of treatment in such grade. Abnormal IO muscle biopsy was taken from a case of enucleation for comparative purpose. The study was reviewed by Al-Azhar university board and didn't require patient's prior acceptance.

\section{Microscopic examination}

All specimens were divided and cut into $1 \mathrm{~mm}^{3}$ cubes. Some cubes were fixed in formaldehyde for examination by the light microscope. Other cubes were fixed in glutaraldehyde and routinely prepared for examination by the electron microscope.

Semi-thin sections $(1 \mu \mathrm{m})$ were cut and prepared by the ultra-microtome then stained with hematoxylin and eosin $(\mathrm{H} \& \mathrm{E})$ stain and with toluidine blue stain to be examined under the light microscope.

Ultra-thin sections $(70 \mathrm{~nm})$ were stained by lead citrate and uranyl acetate and examined by the transmission electron microscope (JEM-1400Plus) at the unit of Electron Microscopy at Faculty of Science, Alexandria University.

\section{RESULTS}

The patients involved in the study ranged from $3-12$ years old with the mean age 6.8 years and standard deviation of 3.2. The total number of cases was six (four females $66.7 \%$ and two males $33.3 \%$.(

\section{Histopathological changes}

Grade II

$\% 60$ of the nerve bundles were hypertrophied. $60 \%$ of the muscle fibers were degenerated. $20 \%$ of the muscle fibers were splitted. The nuclei had large vacuoles and many mitochondria.

Grade III

There were increase in mitochondrial numbers. 10\% of the muscle fibers showed multiple lipid vacuoles (fatty infiltration.(

Infiltration of the muscle fibers by mononuclear cells noticed in $30 \%$ of samples. Endomysium and perimysium were fibrosed in $30 \%$ of samples.
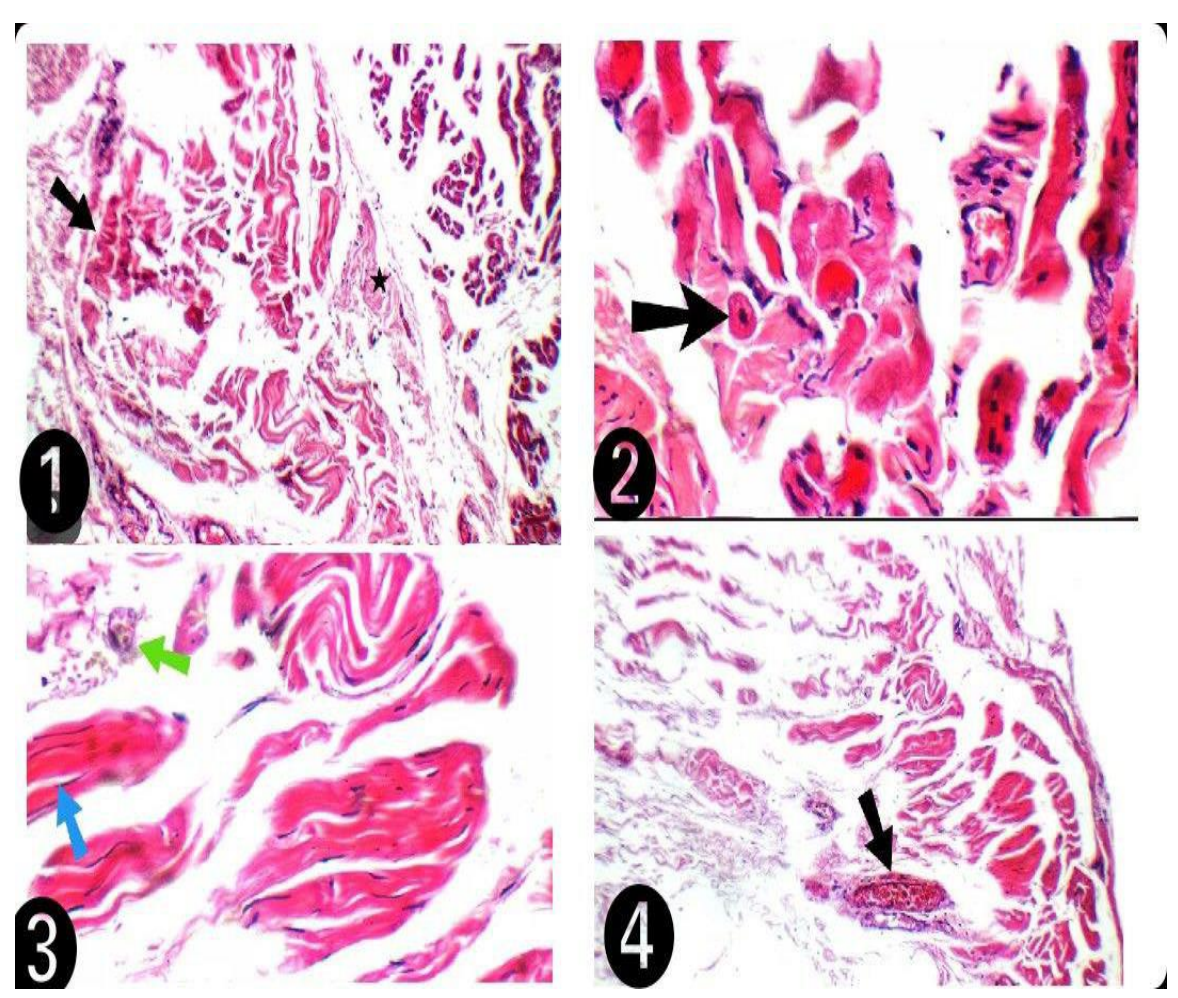

Fig. 1: Haematoxylin and eosin (H\&E) photomicrographs: Photos show variable sizes of muscle fibers (1) Muscle fibers are hypercontracted (black arrow) (2) Regenerating muscle fibers with vesicular nucleus (black arrow) (3) Hypertrophied nerve fiber (green arrow), split muscle fiber (blue arrow) (4) congested blood vessel (black arrow). 
To be noted: (1) \& (4) X200, (2) \& (3) X400. Photomicrographs (1) \& (4) belonged to grade II while (2) \& (3) belonged to grade III.

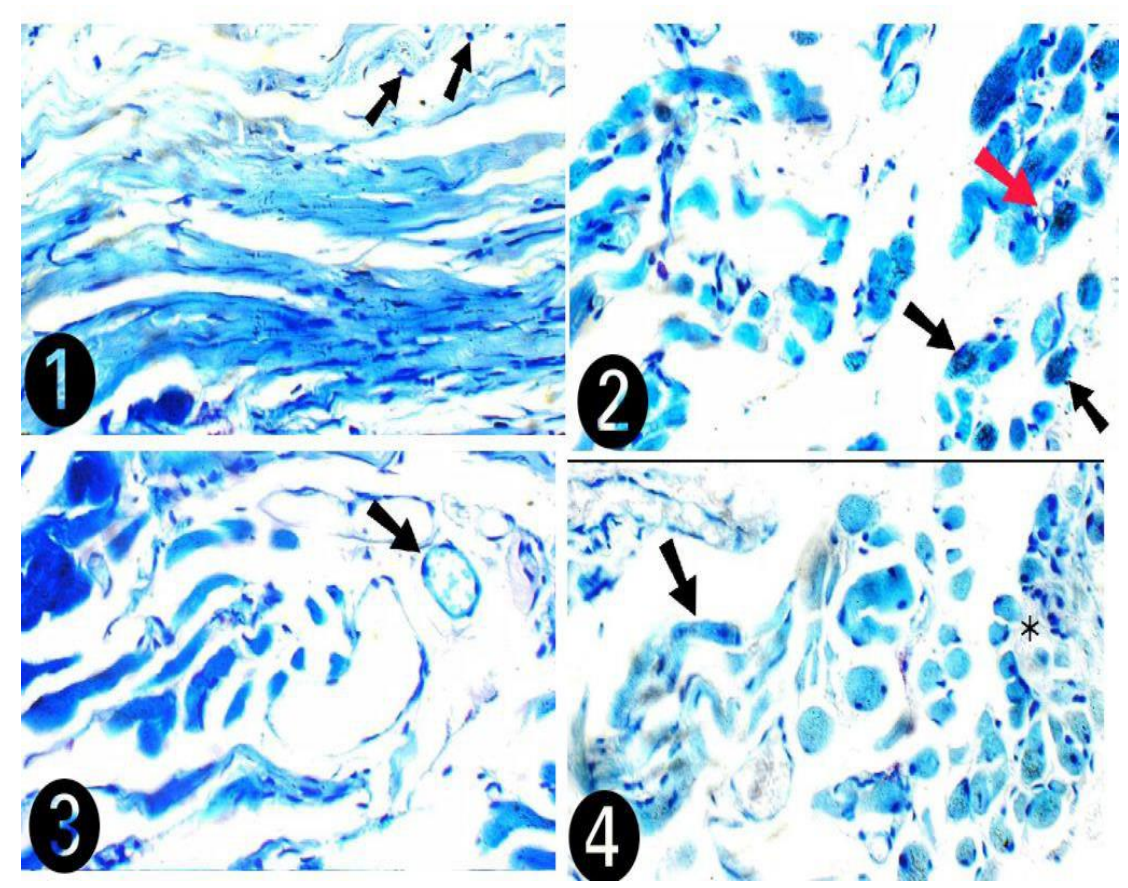

Fig. 2: Toluidine blue photomicrographs: photos show variable sizes of muscle fibers (1) mild mononuclear cellular infiltration (black arrows) (2) cytoplasmic vacuoles (red arrow), inclusion bodies (black arrows) (3) hypertrophied nerve fiber (black arrow) (4) hypercontracted muscle fibers (black arrow) mild endomysial fibrosis $(*)$.

To be noted Toluidine blue X 400. Photomicrographs (1) belonged to grade II while (2), (3) \& (4) belonged to grade III.

\section{Electron microscopic results}

The mitochondria showed mild pleomorphism. Mitochondria accumulated in the subsarcolemmal area and were found to be scattered between myofilaments. Mitochondrial degeneration with vacuolations presented in many samples. Muscle fibers were vacuolated and hypercontracted. Large nucleus and prominent nucleolus were found in many samples. 

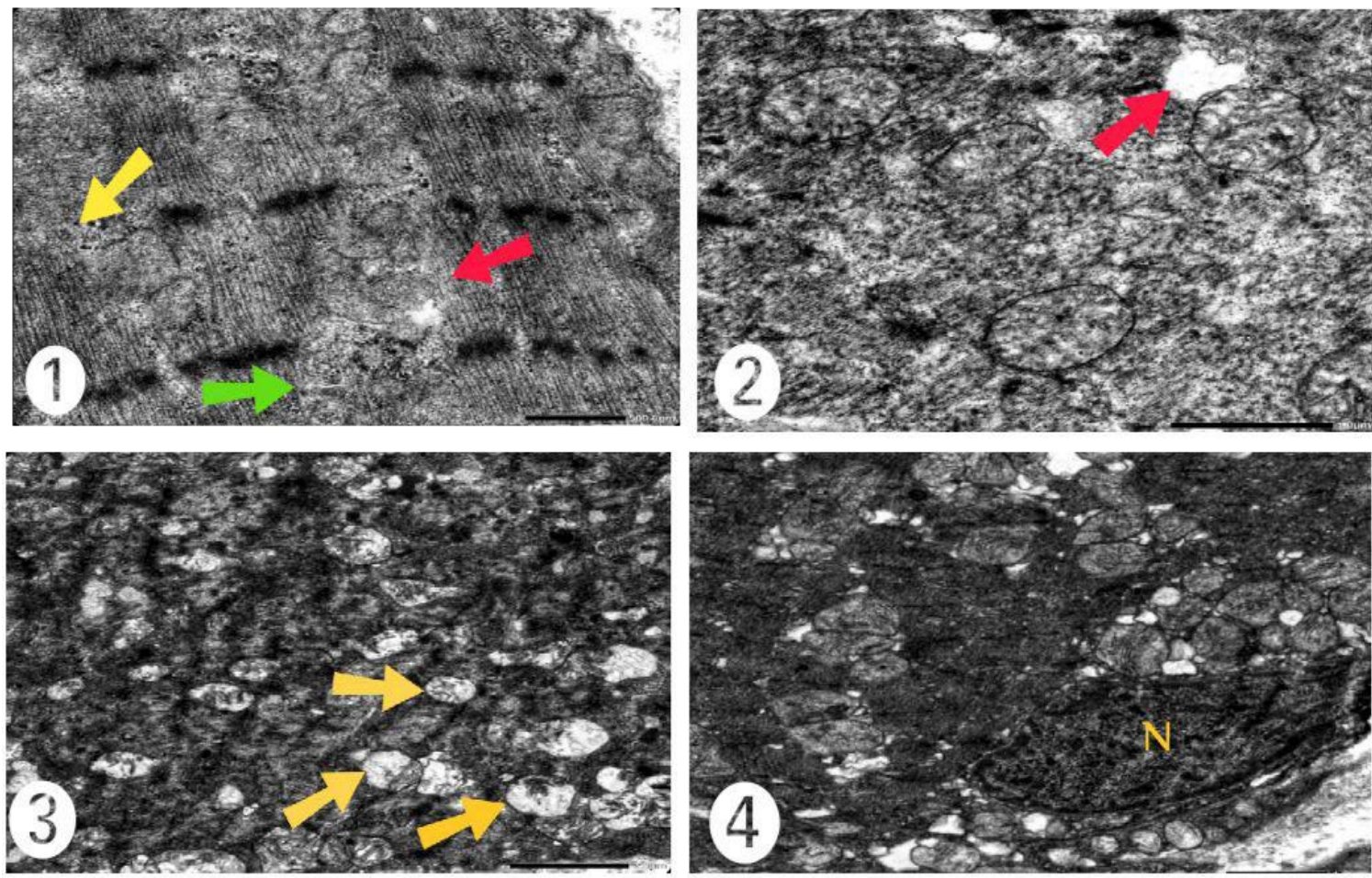

Fig. 3: Electron microscopic photomicrographs: Photos show (1) focally disorganized sarcomere (red arrow), loss of Z-bands (yellow arrows), markedly swollen mitochondria with loss of cristae (green arrow) (2) scattered large vacuoles (red arrow) (3) variable sized markedly swollen mitochondria with marked loss of cristae (yellow arrows) (4) pyknotic peripherally located nucleus (N).

To be noted photomicrographs (1), (2) \& (4) belonged to grade II while (3) belonged to grade III.

\section{DISCUSSION}

Ten muscle biopsies were included in this study, obtained from six patients, four of them had a bilateral muscle affection and two were unilaterally affected. Six biopsies out often $(60 \%)$ belonged to grade II IO overaction, and four biopsies out of ten (40\%) belonged to grade III IO overaction.

All muscle biopsies showed structural changes under light and electron microscopes. As regard muscle fibers, the examination revealed degenerated, regenerating, hyper-contracted, and rounded fibers ${ }^{10}$. Hamdi MM et al agreed with the current study in the presence of subsarcolemmal inclusions of granular fibrillar aggregates ${ }^{6}$.

Also, nuclei appeared large and vesicular. All of the previous findings had been reported by Meyer E, Ludatscher RM and Zonis $\mathrm{S}^{10}$.

Hamdi MM et al announced in their study the same findings that agreed with the current study results. In addition, Choi DG and Chang BL agreed with the current study in prescience of muscle fiber variable sizes. ${ }^{11}$

Vacuoles, especially fat vacuoles, were noted in some cases and this was agreed with Hamdi MM et $\mathrm{al}^{6}$.
Magaudda L et al agreed with the current study that the satellite cells activation is followed by appearance of regenerating muscle fibers. ${ }^{12}$

Hamdi MM et al reported that many of muscle fibers had vacuoles and infiltration by mononuclear cells. Mitochondrial aggregation and pleomorphism were also reported especially in grade III. ${ }^{6}$

\section{CONCLUSION}

This study confirmed the relationship between the clinical grade of the IO muscle overaction and the alternated ultrastructure of the muscle.

Conflict of interest: The authors declared that there are no conflicts of interest.

\section{REFERENCES}

1. Stager D, Dao L and Felius J. Uses of the inferior oblique muscle in strabismus surgery. Middle East African Journal of Ophthalmology. 2015; 22 (3): 292-7.

2. Standring S. Grey's Anatomy: Anatomical Basis of Clinical Practice. (41th ed.), Elsevier; 2016. PP. 63165 . 
3. Demer JL, Oh SY, Clark RA, et al. Evidence for a Pulley of the Inferior Oblique Muscle. Investigative Ophthalmology \& Visual Science. 2003; 44 (9): 3856-62.

4. Modi NC and Jones DH. Strabismus: Background and Surgical Techniques. Journal of Perioperative Practice. 2008; 18 (12): 532-5.

5. Porter JD, Baker RS, Ragusa RJ, et al. Extraocular muscles: Basic and clinical aspects of structure and function. Survey of Ophthalmology. 1995; 39 (6): 451-84.

6. Hamdi MM, El-Hawary GR, El-Hefnawy NG, et al. Histopathological and electron microscopic study for different grades of inferior oblique muscle overaction. Clinical Ophthalmology. 2013; 7 (1): 917-21.

7. Kierszenbaum AL and Tres LL. Histology and cell biology an introduction to pathology. (4th ed.) Elsevier, Canada, 2016; PP. 217-39.

8. Yu Wai Man CY, Chinnery PF, Griffiths PG. Extraocular muscles have fundamentally distinct properties that make them selectively vulnerable to certain disorders. Neuromuscular Disorders. 2005; 15 (1): 17-23.

9. Ruff RL. More than meets the eye: extraocular muscle is very distinct from extremity skeletal muscle. Muscle Nerve. 2002; 25 (3): 311-3.

10. Meyer E, Ludatscher RM, Zonis S. Primary and secondary overacting inferior oblique muscles: an ultrastructural study. British Journal of Ophthalmology. 1984; 68 (6): 416-20.

11. Choi DG, Chang BL. Electron microscopic study on overacting inferior oblique muscles. Korean Journal of Ophthalmology. 1992; 6 (2): 69-75.

12. Magaudda L, Mauro DD, Trimarchi F, et al. Effects of physical exercise on skeletal muscle fiber: ultrastructural and molecular aspects. Basic Applied Myology. 2004; 14 (1): 17-21. 